\title{
Enantioselective Fluoroarylation Catalyzed by Palladium
}

Metal-Catalyzed

Asymmetric

Synthesis and

Stereoselective

Reactions

\section{Key words}

fluoroarylation

styrenes

palladium

\section{SYNFACTRer}<smiles>C=Cc1ccccc1C(=O)N[18F]</smiles>

$\operatorname{ArB}(\mathrm{OH})_{2}$ (2-ethylhexylO) ${ }_{2} \mathrm{PO}_{2} \mathrm{H}(30 \mathrm{~mol} \%)$ Selectfluor (2 equiv) $\underset{\mathrm{CH}_{2} \mathrm{Cl}_{2}-\mathrm{H}_{2} \mathrm{O}(5: 1) \text { r.t. } 15 \mathrm{~h}}{\longrightarrow}$<smiles>O=C(NP)c1ccccc1[C@@H](F)CBr</smiles>

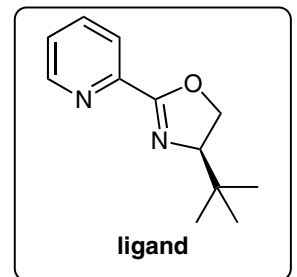

Selected examples:<smiles>COc1ccc(NC(=O)c2ccccc2[C@@H](F)Cc2ccc(C)cc2)cc1</smiles><smiles>[14CH3]</smiles>
$96 \%$ ee<smiles>COc1ccc(NC(=O)c2ccccc2[C@@H](F)Cc2ccc(Cl)cc2)cc1</smiles>

$83 \%$ yield $91 \%$ ee<smiles>COc1ccc(NC(=O)c2ccccc2[C@@H](F)Cc2cccc(F)c2)cc1</smiles>

$$
71 \% \text { yield }
$$
$87 \%$ ee<smiles>COc1ccc(NC(=O)c2ccccc2C(F)Cc2ccccc2)cc1</smiles>

$77 \%$ yield $94 \%$ ee

Possible reaction pathway:

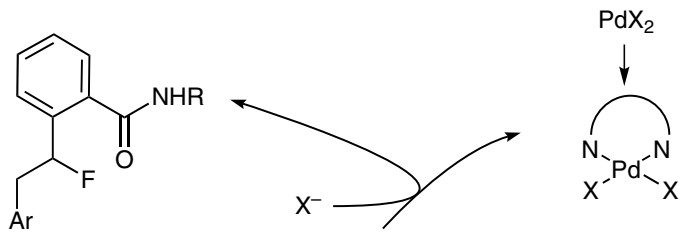

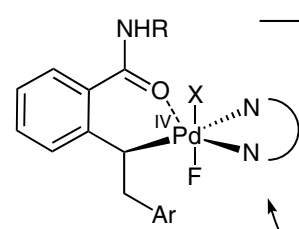<smiles></smiles><smiles>CCC(C)CF</smiles>

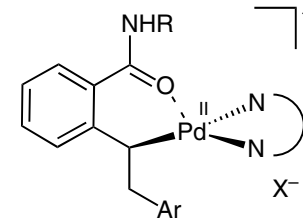<smiles>[Y][Pb]1([Ge])NCCCN1</smiles>

Significance: The authors developed a highly enantioselective palladium-catalyzed fluoroarylation of styrenes bearing an amide-based directing group. The proposed reaction mechanism involves a palladium(IV) intermediate as shown above.

SYNFACTS Contributors: Hisashi Yamamoto, Yasushi Shimoda Synfacts 2014, 10(6), 0617 Published online: 16.05.2014 Dol: 10.1055/s-0033-1339002; Reg-No.: H05214SF
Comment: In this reaction, N,N-ligands play a crucial role to afford the fluoroarylated products. Without these ligands only Heck products were obtained. Organic phosphate was added as phase-transfer catalyst to increase the chemical yield. 